\title{
Estudio de una muestra de pacientes con síndrome de boca ardiente
}

\author{
Rodríguez de Rivera Campillo ME*, López López J**, Chimenos Küstner E**, \\ Sabater Recolons MM***
}

\section{RESUMEN}

El síndrome de boca ardiente (SBA) es una enfermedad que, a pesar de conocerse desde hace muchos años, sigue planteando problemas de diagnóstico y tratamiento. Se manifiesta clínicamente como sensación de ardor o escozor en alguna zona de la mucosa oral, no existiendo ninguna lesión que pueda justificar dicha sintomatología. Se presenta fundamentalmente en mujeres postmenopáusicas, que suelen presentar sus molestias durante periodos prolongados. El ardor bucal puede acompañarse de otras alteraciones sensitivas, como sensación de sequedad o alteraciones gustativas.

Objetivos: Estudiar una muestra de pacientes con SBA y describir los factores epidemiológicos, las enfermedades que padecen y los fármacos que consumen.

Pacientes y métodos: La muestra estaba formada por 83 pacientes que consultaban en la clínica odontológica por ardor bucal. Se efectuó en todos ellos una historia clínica detallada y se registraron todas las características en una hoja de protocolo.

Resultados: El 90,4\% de los pacientes de nuestro estudio eran mujeres y sólo el 9,6\% eran hombres. La media de edad de la muestra fue de 64,9 años. Todos los pacientes presentaban ardor en la lengua y el $64 \%$ lo presentaban en los labios. La mayoría de los pacientes estudiados (76\%) presentaban una evolución de más de 12 meses, mientras que los de menos de 6 meses únicamente llegaban al 6\%. De los 83 pacientes estudiados, 52 presentaban trastornos psicológicos (63\%) y más de la mitad de ellos consumía algún fármaco psicoactivo. Conclusiones: Nuestra muestra de pacientes es equiparable a las descritas en otros estudios. El SBA predomina en mujeres postmenopáusicas, que presentan con gran frecuencia ansiedad y/o depresión.

Palabras clave: Síndrome boca ardiente, glosodinia, boca urente.

\section{SUMMARY}

The burning mouth syndrome (BMS) is known since many years ago, but it is still difficult to be diagnosed and treated. The unique clinical manifestation is a kind of burning sensation on the oral mucosa, without any lesions justifying this symptom. It presents mainly in postmenopausal women, showing their discomfort during long periods. Burning mouth sensation can be accompanied by other sensitive alterations, like dryness or gustative changes.

Objetives: To study a sample of BMS patients, describing the epidemiological factors, their diseases and drug consumption.

Patients and methods: The sample was represented by 83 patients, consulting because their burning mouth sensation. All of them were accurately interrogated and examined and the data were registered in a protocol sheet. Results: $90.4 \%$ of patients were women and $9.6 \%$ were men. The mean of age was $64.9 \%$. All the patients presented burning sensation on the tongue and $64 \%$ on the lips. The majority of the studied patients (76\%) presented an evolution of the symptoms during more than 12 months; only $6 \%$ presented this evolution during less than 6 months. 52 (63\%) of the 83 patients had psychological manifestations and more than the half of them consumed some psychoactive drug. 
Conclusions: Our sample of patients is comparable to those of other studies. BMS is more prevalent in postmenopausal women, presenting frequently anxiety and/or depression.

Key words: Burning mouth syndrome, glossodynia, stinging mouth.

Fecha de recepción: Febrero 2007.

Aceptado para publicación: Febrero 2007.

* Doctora en Medicina y Cirugía, Médico especialista en Dermatología. Odontóloga. Profesora Asociada de Medicina Bucal (UB).

** Doctor en Medicina y Cirugía. Médico especialista en Estomatología. Profesor Titular de Medicina Bucal (UB).

*** Doctora en Medicina y Cirugía. Médico especialista en Estomatología. Profesora Asociada de Medicina Bucal (UB).

Rodríguez de Rivera Campillo ME, López López J, Chimenos Küstner E, Sabater Recolons MM. Estudio de una muestra de pacientes con síndrome de boca ardiente. Av. Odontoestomatol 2007; 23 (3): 141-151.

\section{INTRODUCCIÓN}

El síndrome de boca ardiente (SBA), también llamado glosodinia, glosopirosis, estomatodinia o estomatopirosis es una enfermedad que, a pesar de haber sido descrita desde hace muchos años, sigue planteando aún hoy en día problemas de diagnóstico y tratamiento (1-5). Se caracteriza por la presencia de ardor o escozor en alguna zona de la mucosa oral, especialmente en la lengua y en los labios, no existiendo ningún tipo de lesión que pueda justificar dicha sintomatología. La mayoría de los pacientes son mujeres postmenopáusicas, que suelen presentar un cuadro de ansiedad y/o depresión. Estas pacientes suelen tener un perfil característico: se encuentran entre los 50 y 60 años de edad, padecen estas molestias desde hace bastante tiempo y han sido visitadas y tratadas por diferentes especialistas, sin que se haya resuelto su problema; existe un componente emocional importante y suelen presentar cancerofobia $(4,6)$. El ardor bucal puede acompañarse de otras alteraciones sensitivas, como sensación de sequedad o alteraciones gustativas, como percepción de gusto metálico o amargo $(7,8)$. En algunos casos refieren otras disestesias en la boca, como sensación de tener arenilla o de hinchazón (7-9).

En la actualidad la verdadera etiopatogenia de esta entidad sigue siendo desconocida y esto dificulta el avance en la investigación del tratamiento. De hecho, con ninguno de los tratamientos publicados hasta la actualidad se han obtenido resultados totalmente satisfactorios (10-15). Las últimas investigaciones demuestran mejorías importantes con el empleo de un fármaco, el clonacepam, administrado por vía oral (7) o disuelto en la boca $(16,17)$.

La mayoría de estos pacientes tienen una edad media o avanzada y presentan varias enfermedades sistémicas, por lo que suelen consumir varios fármacos, como hipotensores, diuréticos, fármacos para el aparato digestivo, ansiolíticos o antidepresivos. Muchos de estos fármacos tienen efecto xerostomizante, por lo que pueden agravar la sensación de sequedad $(18,19)$. Sin embargo, la mayoría de estudios que medían la saliva no han podido demostrar alteraciones cuantitativas, aunque sí cualitativas en algunos pacientes $(20,21)$.

El objetivo principal de nuestro trabajo fue estudiar a una población de pacientes con SBA, comparando si los resultados eran equiparables a los obtenidos en otros estudios sobre SBA. Con esa finalidad se investigaron y registraron:

1. La sintomatología que presentaban los pacientes.

2. La localización.

3. El tiempo de evolución. 
4. Las enfermedades sistémicas que padecían y

5. Los fármacos que consumían los pacientes.

\section{METODOLOGÍA}

\section{Pacientes}

La muestra estaba formada por 83 pacientes, que consultaban por síntomas de ardor bucal, desde enero de 2003 a julio de 2006. Algunos de ellos acudían por primera vez a la Clínica Odontológica de la Universidad de Barcelona para realizar tratamiento odontológico; otros solicitaban tratamiento para su patología y en algunos casos eran pacientes remitidos por otros profesionales, que no conseguían mejorar su sintomatología.

Se incluyeron en el estudio todos los pacientes que referían ardor bucal, en ausencia de lesiones orales que lo justificaran, y que llevaran por lo menos un mes sin efectuar ningún tratamiento específico.

Se excluyeron del estudio los pacientes que presentaban algún tipo de lesión en la mucosa oral, los que ya estaban recibiendo tratamiento para el SBA y los que no podían acudir a las visitas del control por problemas personales o médicos. Los pacientes en los que descubrimos en la exploración alguna patología (como úlceras traumáticas, lesiones de liquen plano oral o candidiasis) fueron tratados de su enfermedad y sólo se incluyeron en el estudio si, una vez curadas sus lesiones, persistía la sintomatología.

No se excluyó del estudio ningún paciente que presentase alguna enfermedad sistémica acompañante, ni tampoco los que estaban recibiendo tratamiento para otras alteraciones. Las personas que estaban tomando psicotropos, como ansiolíticos y antidepresivos, no fueron descartadas, al demostrarse en la literatura la gran prevalencia de esta enfermedad en los pacientes con antecedentes psiquiátricos.

\section{MÉTODOS}

Los pacientes fueron visitados en la Unidad de Medicina Bucal de la Clínica Odontológica de la Universidad de Barcelona y en la consulta privada.
Se realizó en todos ellos una historia clínica detallada, insistiendo en los antecedentes patológicos (especialmente psiquiátricos) y en los fármacos que consumían en el momento de la visita o con anterioridad. Se indagó especialmente el consumo de ansiolíticos o antidepresivos. Se realizó una hoja de protocolo donde se recogieron los datos más significativos de la historia clínica, agrupados en 6 apartados:

- Datos de filiación del paciente.

- Antecedentes patológicos, anotando las enfermedades, que, tal como se cita en la literatura, se presentan con mayor frecuencia en estos pacientes: enfermedades cardiocirculatorias, enfermedades del aparato digestivo, patología hormonal y trastornos psiquiátricos.

- Fármacos que estaban tomando los pacientes y duración del tratamiento, agrupándolos en fármacos indicados para una patología cardiorrespiratoria, fármacos que actúan sobre el aparato digestivo, sustitutivos hormonales, hipnóticos, ansiolíticos, antidepresivos, analgésicos, antiinflamatorios, hipolipemiantes, hipoglicemiantes y vitaminas.

- Datos de la exploración bucal: presencia de lesiones en la mucosa oral, caries, enfermedad periodontal y uso de prótesis.

- Datos de laboratorio que aportaba el paciente y que se habían recogido en la historia clínica.

- Datos relacionados con las características clínicas del dolor. Se efectuaron 11 registros sobre las características de la sintomatología (Tabla 1).

\section{RESULTADOS}

Tras efectuar el análisis estadístico de los datos se obtuvieron los resultados que se exponen a continuación. De los 83 pacientes de la muestra, 75 fueron mujeres (el 90,4\%) y únicamente 8 fueron varones (el 9,6\%) (Figura 1).

La media de edad de la muestra fue de 64,9 años; su distribución, categorizada entre los menores de 60 años, entre 60 y 70 años y mayores de 70 años resultó ser la que muestra la figura 2. 


\section{TABLA 1.- FICHA DE PROTOCOLO UTILIZADA EN EL ESTUDIO.}

Ficha

número

fecha

1. Datos de filiación

Apellidos

Nombre

Dirección C.P

Teléfono

Profesión

Sexo

Fecha nacimiento

Procedencia

2. Antecedentes patológicos

3. Fármacos: ansiolíticos, antidepresivos, antihipertensivos, otros

\section{Duración del tratamiento}

4. Exploración bucal

-Aspecto mucosa

-Estado dental/periodontal

-Portador de prótesis

5. Análisis de laboratorio: Rcto y formula GOT ....... Bilirrubina Vita. B12

Glucosa Urea ..... Creatinina GPT saturación de transferrina Zn.....

Ác. Fólico...... Fe ........ Ferritina porcentaje

6. Protocolo para la evaluación del dolor (Woda, Navez, Picard, Gremeau, Pichard-Leandri, 1998) (Se detalla en la Tabla 3)

7. Escala del dolor (valorada de 0 a 10 )

8. Seguimiento (Respuesta al tratamiento)

\begin{tabular}{|l|l|l|l|l|l|l|}
\hline Fecha & \multicolumn{2}{|l|}{ Clonacepan } & Placebo & \multicolumn{2}{l|}{} \\
\hline & Peor & Mejor & Igual & Peor & Igual & Mejor \\
\hline & & & & & & \\
\hline & & & & & & \\
\hline & & & & & & \\
\hline & & & & & & \\
\hline
\end{tabular}

Barcelona, de de 200

Firma del paciente 


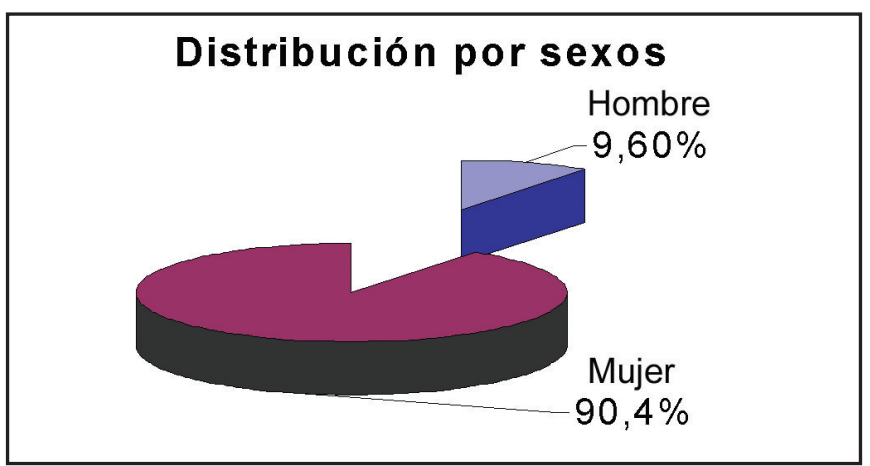

Fig. 1. Distribución por sexos de la muestra (75 mujeres y 8 varones).

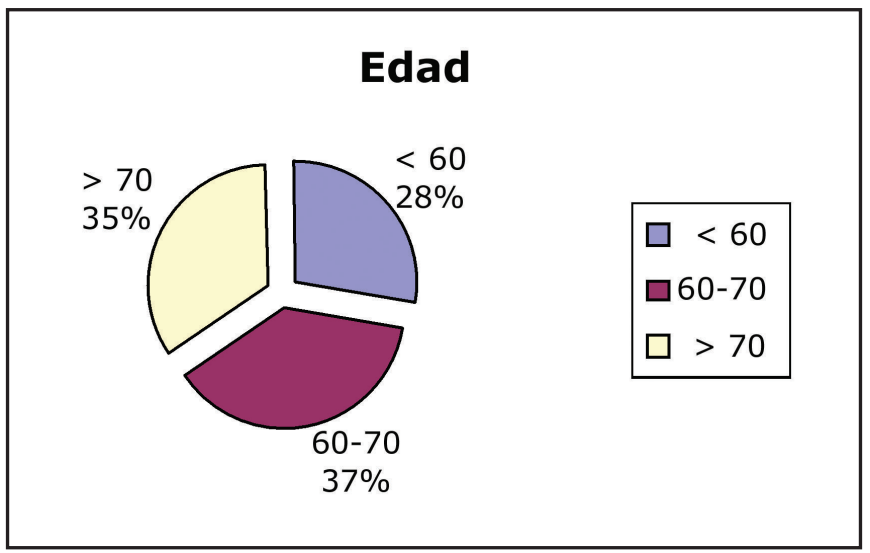

Fig. 2. Distribución por edades.

En lo que respecta a la presencia de enfermedades sistémicas, de los 83 pacientes, 52 de ellos (el 63\%) presentaban trastornos psicológicos, mientras que 31 (el 37\%) no padecían alteraciones psicológicas. Cerca del $40 \%$ de los pacientes presentaban alguna enfermedad hormonal. En el $47 \%$ de ellos se había diagnosticado alguna patología cardiovascular y el $54 \%$ de los pacientes padecían algún tipo de patología gastrointestinal (Figura 3).

Tal como se puede comprobar en la figura 4, uno de cada cuatro pacientes (el 25,3\%) presentaba lesiones de la mucosa oral (LMO), el 26,5\% presentaron patología dental y periodontal (PDP) y más de la mitad (53\%) utilizaban prótesis.

Del total de los pacientes, 72 (87\%) presentaban un dolor simétrico, mientras que únicamente el $13 \%$ presentaban una marcada asimetría en el dolor.

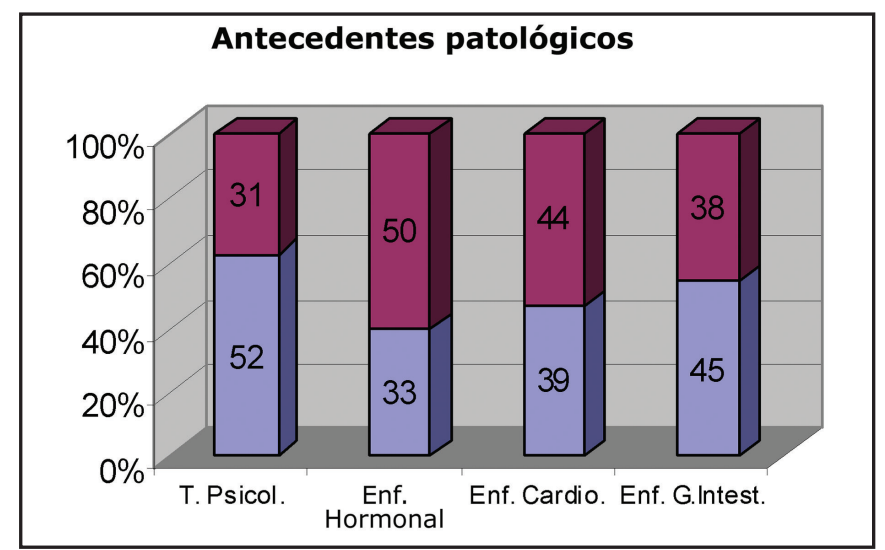

Fig. 3. Distribución en función de las enfermedades sistémicas.

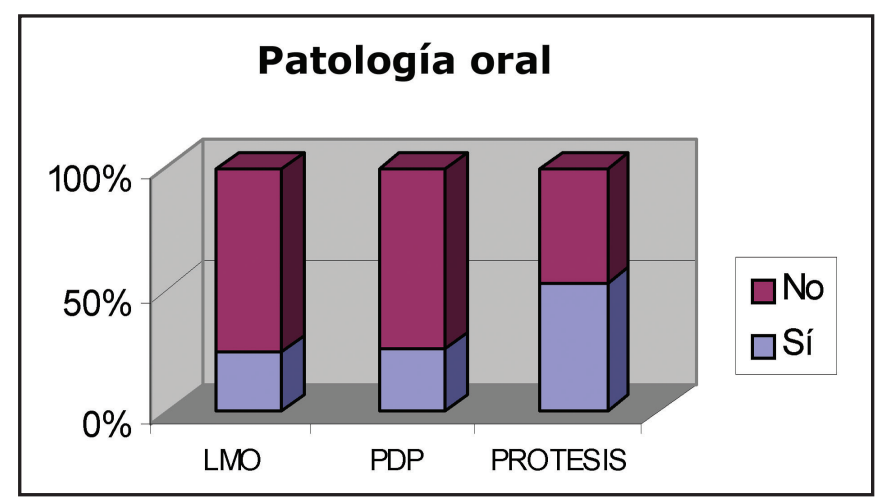

Fig. 4. Distribución en función de la patología oral.

Se preguntó a los pacientes por los factores que coincidieron con el inicio de la enfermedad, incidiendo fundamentalmente sobre el estrés o algún tratamiento dental. Menos del 30\% de los pacientes reportaron inicio de la enfermedad debido al estrés y algo menos del $20 \%$ debido a algún tratamiento odontológico. El resto no recordaban bajo qué circunstancias se había desencadenado la sintomatología.

Más de la mitad de los pacientes referían que las molestias se agravaban tras un periodo de tensión emocional. Así mismo la masticación de algún tipo de alimento actuaba como factor desencadenante en más del $50 \%$ de los pacientes y la temperatura muy extrema de los alimentos empeoraba la sintomatología en el $40 \%$ de los pacientes.

Se estudiaron la aparición de otros dolores en diferentes localizaciones que pudieran tener algún tipo 
de relación con el valorado en el estudio. Observamos que más de la mitad de los pacientes presentaban mialgias, algo más del 20\% (22 personas) tenían molestias oculares, y, únicamente 5 de 75 mujeres presentaban molestias vaginales.

El dolor orofacial más común entre los pacientes estudiados fue la cefalea tensional, presente en un $65 \%$.

Más de la mitad de los pacientes de nuestro estudio consumía algún tipo de fármaco psicoactivo, llegando casi al $75 \%$ en el caso de los ansiolíticos.

Otros fármacos consumidos con gran frecuencia eran los destinados al aparato digestivo, que tomaban más de la mitad de los pacientes, analgésicos o antiinflamatorios $(45,8 \%)$ y fármacos para el aparato cardiovascular (40\%).

En lo que respecta a las características clínicas, todos los pacientes referían ardor y el $88 \%$ sentían sequedad. La sensación de hinchazón y granular o de arenilla eran referidas con menor frecuencia.

La totalidad de los pacientes de nuestra muestra presentaban ardor en la lengua, el $64 \%$ lo presentaban también en los labios, especialmente en el inferior y más de la mitad en el paladar. La encía y la orofaringe fueron localizaciones menos frecuentes (Figura 5).

El tiempo de evolución de la enfermedad se categorizó en: menos de 6 meses, entre 6 y 12 meses, o

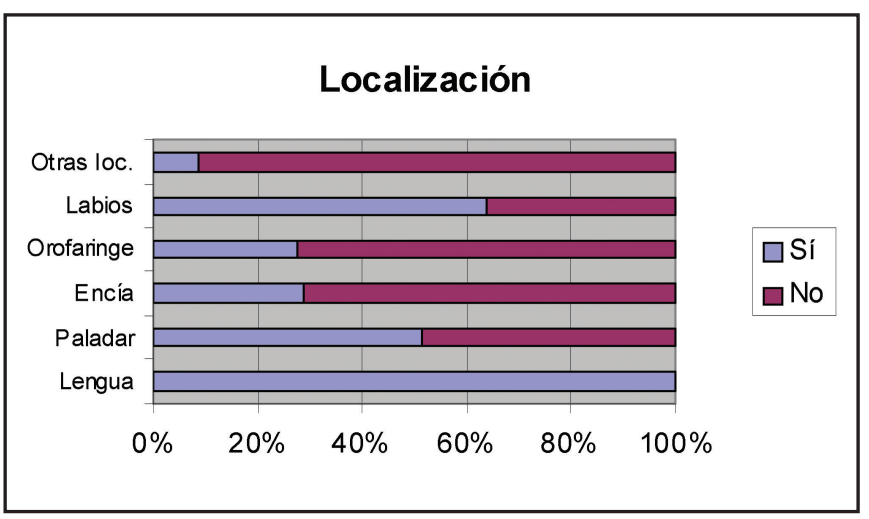

Fig. 5. Distribución según la localización del dolor. más de 12 meses. La mayoría de los pacientes estudiados (76\%) presentaban una evolución de más de 12 meses, mientras que los de menos de 6 meses únicamente llegaban al 6\% (Figura 6).

\section{DISCUSIÓN}

Del total de la muestra estudiada, 83 pacientes, 75 $(90,4 \%)$ fueron mujeres y sólo $8(9,6 \%)$ fueron varones. Estos datos coinciden con los obtenidos por otros autores en estudios similares, en los que refieren que existe una marcada predilección por el sexo femenino $(4,6,22)$. Silvestre, Bagán y Rojo (23), en un trabajo realizado en 1991 , sobre 110 pacientes, hallaron una proporción de 8/1. Gorsky y cols. (12), de un total de 130 pacientes, 101 eran mujeres y 29 eran hombres.

La media de edad de la muestra fue de 64,9 años, variando desde los 36 a los 86 años. Estos resultados son comparables a los obtenidos en otros estudios, como el de Ship y Grushka (24), en el que la edad media fue de 50 a 60 años, con un rango comprendido entre los 36 y 84 años. También obtuvieron resultados similares otros autores, como Bergdahl y Zakrzewska (25-27).

Hay que destacar el gran porcentaje de enfermedades sistémicas que padecía nuestro grupo de pacientes afecto de SBA. El 63\% de la muestra (52 pacientes) presentaba trastornos psicológicos, entre los que predominaban estados de ansiedad, depresión o alteraciones del sueño. Ship y cols. (24), en

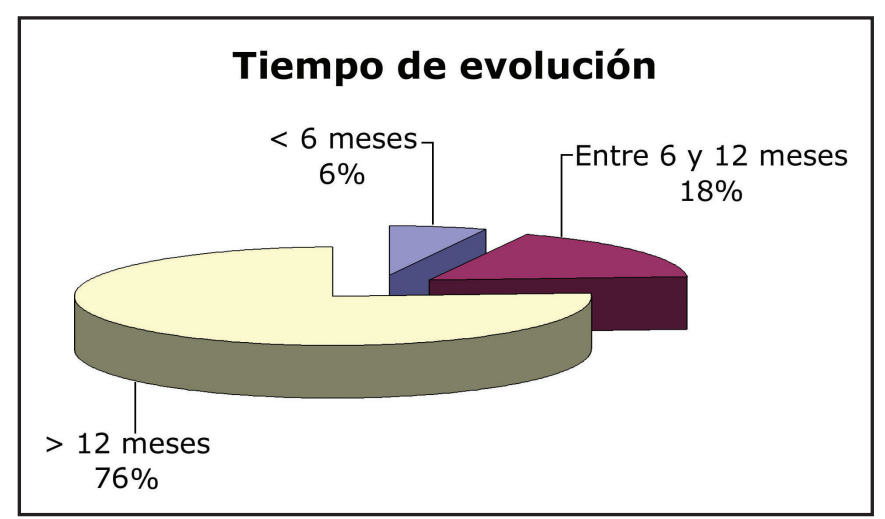

Fig. 6. Distribución según el tiempo de evolución. 
un estudio efectuado en 1995, referían que en un 51,3\% de los pacientes con SBA se podía establecer un diagnóstico psiquiátrico. En un estudio posterior, Bogetto y cols. (28) relataban que el $69,4 \%$ de sus pacientes habían padecido algún trastorno psiquiátrico con anterioridad, siendo los trastornos más frecuentes la depresión y la ansiedad. El 60\% de los pacientes padecían alguna enfermedad hormonal, entre las que se consideraron las alteraciones de tiroides, los déficits de hormona femenina provocados por menopausia precoz u ooforectomía y la diabetes, tanto la de tipo I como la de tipo II. Otros autores, como Carrington y Getter $(29,30)$, también encontraron mayor incidencia de diabetes entre sus pacientes.

El 54\% de los pacientes referían algún tipo de patología gastrointestinal, entre las que se consideraban gastritis, hernia de hiato, ulcus péptico y colon irritable. Muchos de estos pacientes estaban recibiendo tratamiento con antiulcerosos, protectores gástricos y fármacos para reducir las flatulencias y habían sido sometidos a pruebas endoscópicas. Otros estudios, como el de Soto y cols. (31), también demuestran que estos pacientes tienen mayor tendencia a presentar enfermedades de base psicosomática, como trastornos digestivos, liquen plano oral y estomatitis aftosa recurrente.

El 47\% de ellos presentaba alguna enfermedad cardiovascular y estaban siendo tratados con diuréticos, vasodilatadores o betabloqueantes, bien porque presentaban hipertensión arterial o algún otro problema cardíaco o circulatorio. En el estudio de Fleury (32) realizado en 1990 sobre 114 pacientes afectos de SBA, el $18 \%$ padecía enfermedades vasculares.

Estos resultados obtenidos en nuestros pacientes coinciden con los de estudios anteriores (33), en los que se demuestra que los pacientes con SBA padecen numerosas enfermedades sistémicas y consumen un gran número de medicamentos.

Hay que tener en cuenta que, como en otros estudios, la media de edad de la población era elevada y, por tanto, era de esperar que presentasen una o varias enfermedades sistémicas asociadas (34-36).

Por otra parte, hay que considerar la importancia del consumo de ciertos fármacos como mecanismo etio- patogénico del SBA. Los antidepresivos y ansiolíticos producen xerostomía y pueden desencadenar o agravar los síntomas. Los diuréticos condicionan un estado de depleción de mayor cantidad de líquidos y de iones, hecho que puede agravar también la xerostomía.

Otro hecho a destacar es que el 25,4\% de los pacientes presentaban algún tipo de lesión en la mucosa oral cuando fueron explorados en la primera visita. Algunos presentaban una estomatitis protésica o una candidiasis subplaca, que mejoraba al higienizar la prótesis y al efectuar el tratamiento oportuno. Otros presentaban úlceras traumáticas ocasionadas por el roce de prótesis, que mejoraban al ser retirada o al ser pulida. Algunos pacientes presentaban lesiones en la mucosa yugal, debido al hábito de mordisqueo de dicha mucosa, y lesiones eritematosas en la punta de la lengua, asociadas al hábito de empuje lingual. Otros tenían marcadas las improntas dentales en todo el borde de la lengua, a pesar de que no fueran conscientes del hábito de apretamiento. A estos pacientes se les informó de la necesidad de corregir dichos hábitos, antes de iniciar el tratamiento sintomático.

El 26,5\% de los pacientes presentaba algún tipo de lesión dental o periodontal en el momento de la exploración y el 53\% de ellos llevaba algún tipo de prótesis dental. Resultados similares obtuvieron otros autores como Fleury y cols. (32).

En el $87 \%$ de los pacientes las molestias eran simétricas. Sólo el 13\% de los pacientes presentaban una marcada asimetría en la sintomatología. Si bien no hemos encontrado otros estudios en los que se mida este parámetro, nuestra interpretación clínica orienta hacia una posible neuropatía periférica localizada, en asociación con una intervención odontológica previa (exodoncia, colocación de implantes, etc).

Más de la mitad de los pacientes relacionaban el inicio de las molestias tras un periodo de estrés; estos datos coinciden con los obtenidos en otros estudios $(37,38)$.

La ingesta de bebidas o alimentos muy calientes o muy fríos, también agravaba o calmaba los síntomas 
en más del $40 \%$ de los pacientes, tal como citan otros autores en la literatura $(5,6)$.

Cerca de un $30 \%$ de los pacientes recordaban que el inicio de la sintomatología había coincidido con un periodo de estrés o de máxima preocupación. En cerca de un $30 \%$ de los casos lo relacionaban con algún tratamiento dental recibido. Estos datos coinciden con los que encontramos en otros estudios (4,6,37-39), en los que describen como factores desencadenantes de SBA los períodos de sobrecarga emocional y los tratamientos odontológicos.

Más de la mitad de los pacientes presentaban mialgias y algo más del $20 \%$ tenían molestias oculares. Este alto porcentaje de dolores musculares puede justificarse pensando que se trata de una población de cierta edad que suele presentar enfermedades óseas degenerativas. También hay que pensar que las molestias oculares pueden estar ocasionadas por los cambios que se producen en las mucosas, tanto oral como ocular y vaginal, debido a las alteraciones hormonales producidas durante el climaterio $(40,41)$.

De los 83 pacientes de nuestro estudio, más del $50 \%$ consumían fármacos psicoactivos, entre los que consideramos los antidepresivos, los ansiolíticos y los hipnóticos. Estos datos coinciden con los resultados obtenidos en otros estudios, en los que se demuestran cifras muy altas de trastornos psiquiátricos en estos pacientes $(28,38,42-44)$. De hecho, llama la atención que casi el $75 \%$ de nuestros pacientes eran consumidores habituales u ocasionales de ansiolíticos.

Otros fármacos que consumían los pacientes de nuestro estudio eran fármacos para el aparato digestivo, que consumían más de la mitad de los pacientes, analgésicos o antiinflamatorios (el 45,8\%) y fármacos para el aparato cardiovascular (más del $40 \%$ ). Cifras similares obtienen otros autores en sus trabajos $(12,45,46)$.

El 88\% de los pacientes referían junto al ardor sensación de sequedad, a pesar de que en la mayoría de los casos no se objetivaba en la exploración déficit de saliva. Este dato coincide con lo publicado por Grushka, quien refiere que, junto a la sensación de ardor bucal, muchos pacientes afectos de SBA manifiestan una sensación intensa y desagradable de sequedad bucal, sin que se evidencie clínicamente un déficit de saliva (47). Esta autora describió que existen relaciones anatómicas entre las estructuras responsables de la percepción del gusto, de la sensación de sequedad y del dolor oral. La sensación gustativa es captada por las papilas fungiformes, que se encuentran en los 2/3 anteriores de la lengua. Cada papila fungiforme contiene por término medio 6 receptores gustativos y cada receptor está rodeado por un conjunto de terminaciones sensitivas responsables de la percepción del dolor. Por tanto, las personas "supercatadoras", que son más frecuentes entre las mujeres, son más susceptibles también de padecer dolor oral.

De las 6 posibles localizaciones que se investigaron, se presentó ardor en la lengua en la totalidad de los pacientes. El $64 \%$ refirieron sintomatología en los labios, especialmente el inferior, más del $50 \%$ en paladar y el $30 \%$ en la encía y en la orofaringe. Estos datos coinciden con los de muchos otros autores (48-53).

La mayoría de los pacientes estudiados (76\%) llevaban más de un año padeciendo las molestias; sólo el $6 \%$ de los pacientes sufrían ardor bucal desde hacía menos de 6 meses y el otro $18 \%$ presentaban la sintomatología desde un periodo comprendido entre los 6 y los 12 meses. Resultados similares han sido descritos por otros autores en la literatura, que definen el SBA como un dolor crónico, que llega a hacerse insoportable para el paciente a pesar de llevar largo tiempo sufriendo sus molestias (54-57).

\section{CONCLUSIONES}

Los resultados obtenidos en nuestro estudio son los que se exponen a continuación:

1. Todos los pacientes presentaban ardor en la lengua, y la mayoría referían sequedad oral.

2. La localización más frecuente del ardor fue en la lengua, seguida de los labios, especialmente el inferior.

3. La mayoría de los pacientes padecían SBA desde hacía más de un año. 
4. Las enfermedades sistémicas que presentaban con mayor frecuencia nuestros pacientes fueron los trastornos psicológicos, las enfermedades cardiovasculares, gastrointestinales y hormonales.

5. Los fármacos que consumían con mayor frecuencia eran los psicotropos, los fármacos para el aparato digestivo y para el aparato cardiovascular.

Estos resultados son equiparables a los descritos en la mayoría de estudios en la literatura. Por tanto, podemos concluir que nuestra muestra es totalmente representativa de pacientes con SBA.

\section{BIBLIOGRAFÍA}

1. Santoro V, Caputo G, Peluso F. Clinical and therapeutic experience in twenty eight patients with burning mouth syndrome. Minerva Stomatol 2005;54:489-96.

2. Lamey PJ, Murria BM, Eddie SA, Freeman RE. The secretion of parotid saliva as stimulated by $10 \%$ citric acid is not related to precipitating factors in burning mouth syndrome. J Oral Pathol Med 2001;30:121-4.

3. Klausner JJ. Epidemiology of chronic facial pain:diagnostic usefulness in patient care, J Am Dent Assoc 1994;125:1604-11.

4. Eguía A, Aguirre JM, Etxebarria M, Martínez-Conde R. Síndrome de boca ardiente:aspectos epidemiológicos, etiopatogénicos, clínicos y terapéuticos. Rev Vasca Odontoestomatol 1999;3: 28-41.

5. Sardella A, Lodi G, Demarosi F, Bez C, Cassano S, Carrassi A. Burning mouth syndrome: a retrospective study investigating spontaneous remission and response to treatments. Oral Diseases 2006;12:152-5.

6. Silvestre FJ, Serrano C. El síndrome de boca ardiente:revisión de conceptos y puesta al día. Medicina Oral 1997;2:30-8.

7. Grushka M, Epstein J, Mott A. An open-label, dose escalation pilot study of the effect of clonazepan in burning mouth syndrome. Oral Surg Oral Med Oral Pathol 1998:86 557-61.

8. Grinspin D, Blanco GF, Allevato MA, Stengel FM. Burning mouth syndrome. Int J Dermatol 1995; 34:483-7.

9. Brailo V, Vuêiaeeviae-Boras V, Alajbeg I, Lukenda $\mathrm{J}$, Aeurkoviae M. Oral burning symptoms and burning mouth syndrome-significance of different variables in 150 patients. Med Oral Patol Oral Cir Bucal 2006; 11:E252-5.

10. Epstein JB, Marcoe JH. Topical application of Capsaicin for the treatment of oral neuropathic pain and trigeminal neuralgia. Oral Surg Oral Med Oral Pathol 1994;77:135-40.

11. Petruzzi M, Lauritano D, De Beneditis M, Baldoni M, Serpico R. Systemic capsaicin for burning mouth syndrome:short-term results of a pilot study. J Oral Pathol Med 2004;33:11-4.

12. Gorsky M, Silverman S, Chinn H. Clinical characteristics and management outcome in the burning mouth syndrome. An open study of 130 patients. Oral Surg Oral Med Oral Pathol 1991; 72:192-5.

13. Femiano F, Gombos F, Scully C, Busciolano M, Luca P. Burning mouth syndrome (BMS): controlled open trial of the eficacy of alpha-lipoic (thioctic acid) on symptomatology. Oral Diseases 2000;6:274-7.

14. Femiano F, Gombos F, Scully C. Síndrome de boca ardiente:Estudio de la psicoterapia, medicación con ácido alfa-lipoico y combinación de terapias. Medicina Oral 2004;1:8-13.

15. Eguía A, Aguirre JM, Sagastra O. Tratamiento del Síndrome de Boca Ardiente:Análisis crítico de la literatura y propuesta de un protocolo. Revista Vasca Odontoestomatol 2002;12:24-30.

16. Woda A, Navez ML, Picard P, Gremeau C, PichardLeandri E. A possible therapeutic solution for stomatodynia (burning mouth syndrome). J Orofacial Pain 1998;122:272-7. 
17. Gremeau-Richard C, Woda A, Navez ML, Attal N, Bouhassira D, Gagnieu MC. Topical clonazepam in stomatodynia :a randomised placebocontrolled study. Pain 2004;108:51-7.

18. Marques Soares MS, Chimenos Küstner E, Subirá Pifarré C, Rodríguez de Rivera Campillo E, López López J. Association of Burning Mouth Syndrome with xerostomia and medicines. Med Oral 2005; 10:301-8.

19. Bergdahl M, Bergdahl J. Low unstimulated salivary flow and subjective oral dryness: associaton with medication, anxiety, depression, and stress. J Dent Res 2000;79:1652-8.

20. Nagler RM, Hershkovich O. Age-related changes in unstimulated salivary function and composition and its relation to medications and oral sensorial complaints. Aging Clin Exp Res 2005; 17:35866.

21. Tammiala-Salonen T, Söderling E. Protein composition, adhesion, and agglutination properties of saliva in burning mouth syndrome. Scand J Dent Res 1993;101:215-8.

22. Muzyka BC, De Rossi SS. A review of Burning Mouth Syndrome. Cutis 1999;64:29-35.

23. Silvestre FJ, Bagán JV, Rojo L. Síndrome de boca ardiente:Estudio clínico y biológico. Rev Actual Odontoestomatol Esp 1991;409:37-42.

24. Ship JA, Grushka M, Lipton JA, Mott AE, Sessle BJ, Dionea RA. Burning mouth syndrome:an update. J Am Dent Assoc 1995;126:842-53.

25. Bergdahl J, Anneroth G. Burning mouth syndrome: literature review and model for research and management. J Oral Pathol Med 1993;22: 433-8.

26. Zakrzewska JM. The burning mouth syndrome remains an enigma. Pain 1995;253-7.

27. Bergdahl M, Bergdahl J. Burning mouth syndrome: prevalence and associated factors. J Oral Pathol Med 1999;28:350-4.
28. Bogetto F, Maina G, Ferro G, Carbone M, Gandolfo S. Psychiatric comorbidity in patients with burning mouth syndrome. Psychosomatic Med 1998;60:378-85.

29. Carrington J, Getter L, Brown RS. La glosodinia como forma de presentación de la neuropatía diabética. JADA 2002;6:15-7.

30. Carrington J, Getter L, Brown RS. Diabetic neuropathy masquering as glossodinia JADA 2001;132:1549-51.

31. Soto Araya M, Rojas Alcayaga G, Esguep A. Association beyween psychological disorders and presence of lichen planus, burning mouth syndrome and recurrent aphtous stomatitis. Med Oral 2002;9:1-7.

32. Fleury JE, Deboets D, Assaad C, Maffre N, Duboc B, Ferrey G. Les glossodynies. Rev Stomatol Chir maxillofac 1990;91:276-80.

33. Chimenos E, Marques MS. Boca ardiente y saliva. Medicina Oral 2002;7:244-53.

34. Velasco E, Valencia S, Blanco A, Velasco C. El síndrome de ardor bucal en el anciano. La identificación de los trastornos psíquicos en su etiopatogenia. Rev Esp Geriatr Gerodontol 1998;33:19-24.

35. Lauritano D, Spadari F, Formaglio F, Zambellini AM, Salvato A. Ethiopathogenic, clinical-diagnostic and therapeutic aspects of the burning mouth syndrome. Minerva Stomatol 1998;47: 239-51.

36. Drage LA, Rogers RS. Burning mouth syndrome. Dermatol Clin 2003;21:135-45.

37. Eli I, Kleinhanz M, Baht R, Littner M. Antecedents of burning mouth syndrome (Glossodinia)-Recent life events vs Psychological aspects. J Dent Res 1994;73:567-72.

38. Rojo I, Silvestre FJ, Bagán JV, De Vicente T. Prevalence of psychopatology in burning mouth syndrome. A comparative study among patients with and without psychiatric disorders and 
controls. Oral Surg Oral Med Oral Pathol 1994; 78:312-6.

39. Maresky IS, Van der Bijil P, Gird I. Burning mouth syndrome. Evaluation of multiple variables among 85 patients. Oral Surg Oral Med Oral Pathol 1993;75:303-7.

40. Frutos R, Rodríguez S, Miralles L, Machuca G. Manifestaciones orales y manejo odontológico durante la menopausia. Medicina Oral 2002;7:26-35.

41. Forabosco A, Cricuolo M, Coukos G, Uccelli E, Weinstein R, Spinato S. Efficacy of hormone replacement therapy in postmenopausal women with oral disconfort. Oral Surg Oral Med Oral Pathol 1992;73:570-4.

42. Bergdahl J, Anneroth G, Perris H. Personality characteristics of patients with resistant BMS. Acta Odontol Scand 1995;53:7-11.

43. Somacarrera ML, Pinos HP, Hernández G, Lucas ML. Síndrome de la boca ardiente. Aspectos clínicos y perfil psicológico asociado. Arch Odontoestomatol 1998;14:299-306.

44. Pokupec-Gruden JS, Cekic-Arambasin A, Gruden V. Psycogenic factors in the etiology of stomatopyrosis. Coll Antropol 2000;24:119-26.

45. Bergdahl J, Bergdahl M. Environmental illness: evaluation of salivary flow, symptoms, diseases, medications, and psycological factors. Acta Odontol Scand 2001;59:104-10.

46. Bergdahl M. Salivary flow and oral complaints in adult dental patients. Community Dent Oral Epidemiol 2000;28:59-66

47. Grushka M, Bartashuk LM. Burning mouth syndrome and oral dysesthesias. Oral Health $\mathrm{J}$ 2001;27-33.
48. Eguía-Del-Valle A, Aguirre JM, Martínez-Conde $\mathrm{R}$, Echevarria MA. Síndrome de boca ardiente en el País Vasco:estudio preliminar de 30 casos. Med Oral 2003;8:84-90.

49. Huang W, Rothe MJ, Gran-Kels JM. The burning mouth syndrome. J Am Acad Dermatol 1996;34: 91-8.

50. Lamey PJ. Burning mouth syndrome. Dermatol Clin 1996;14:339-54.

51. López C, Martínez JM, Gómez R, Leco I, Donado M. Estudio clínico-epidemiológico sobre el síndrome de ardor bucal. Avances Odontoestomatol 2003;4:185-91.

52. Gorsky M, Silverman S, Chinn H. Clinical characteristics and management outcome in the burning mouth syndrome. An open study of 130 patients. Oral Surg Oral Med Oral Pathol 1991; 72:192-5.

53. Miyamoto SA, Ziccardi VB. Burning mouth syndrome. Mt Sinai J Med 1998;65:343-7.

54. Woda A, Gremeau-Richard C. Le point sur la stomatodynie. Actualités Odonto-Stomatologiques 1998;204:509-15.

55. Velasco E, Valencia S, Blanco A, Velasco C. El síndrome de ardor bucal en el anciano. La identificación de los trastornos psíquicos en su etiopatogenia. Rev Esp Geriatr Gerodontol 1998; 33:19-24.

56. Grushka M, Epstein JB, Gorsky M. Burning mouth syndrome. Am Fam Physician 2002;15: $615-20$.

57. Woda A, Plonchon P, A unified concept of idiopathic orofacial pain:clinical features. J Oral Pain 1999;13:173-95. 